Romero Jiménez, W., Uribe Torres, G. A., Rojas-Berrio, S., y Robayo-Pinzón, O. (mayo-agosto, 2021). Evaluación de los atributos de las Lovemarks en universidades en Bogotá. Revista Virtual

Universidad Católica del Norte, (63), 223-244. https://www.doi.org/10.35575/rvucn.n63a9

\title{
Evaluación de los atributos de las Lovemarks en universidades en Bogotá
}

Lovemarks attributes in Bogota's universities an evaluation

\section{Wilfredo Romero Jiménez}

Magister en Gerencia Estratégica de Mercadeo

Corporación Universitaria Minuto de Dios

Bogotá, Colombia

wilfred_romero@yahoo.es

Orcid: https://orcid.org/0000-0003-0203-416X

Cvlac: https://scienti.minciencias.gov.co/cvlac/visualizador/generarCurriculoCv.do?cod rh=0000071805

\section{Giomar Alexander Uribe Torres}

Magister en Gerencia Estratégica de Mercadeo

Institución Universitaria Politécnico Grancolombiano

Bogotá, Colombia

giomaruribe@gmail.com

Orcid: https://orcid.org/0000-0003-2484-2651

Cvlac: https://scienti.minciencias.gov.co/cvlac/visualizador/generarCurriculoCv.do?cod_rh=0001767136

\section{Sandra Rojas-Berrio}

Doctora en Ciencias Administrativas

Universidad Nacional de Colombia

Bogotá, Colombia

sprojasb@unal.edu.co

Orcid: https://orcid.org/0000-0002-1148-3779

Cvlac:

http://scienti.colciencias.gov.co:8081/cvlac/visualizador/generarCurriculoCv.do?cod_rh=0000788970

\section{Oscar Robayo-Pinzón}

Magíster en Psicología del Consumidor

Institución Universitaria Politécnico Grancolombiano

Bogotá, Colombia

osrobayo@poligran.edu.co

Orcid: https://orcid.org/0000-0002-1475-2460

Cvlac: https://scienti.minciencias.gov.co/cvlac/visualizador/generarCurriculoCv.do?cod_rh=0000446769 
Recibido: 12 de junio de 2020

Evaluado: 04 de septiembre de 2020

Aprobado: 19 de febrero de 2021

Tipo de artículo: Investigación Científica y Tecnológica

\title{
Resumen
}

Lovemarks es un concepto relevante en el ámbito del mercadeo, y por ello es importante estudiarlo en diversos contextos. En el presente tiene por objetivo la evaluación de los atributos de las Lovemarks en el contexto universitario en Bogotá. Para, ello se utilizó un enfoque deductivo, bajo una modalidad de investigación cuantitativa. La población estuvo representada por 198 estudiantes, 9 administrativos, 9 docentes y 25 graduados de diferentes universidades de Bogotá, y como instrumento de recolección de datos se empleó una encuesta de preguntas abiertas y cerradas. Dicha encuesta, demostró que el atributo "respeto", obtuvo un Path coefficient más favorable, con respecto al "amor"; seguido de "intimidad", "misterio" y, finalmente, "sensualidad". Concluyendo que, las Lovemarks se encuentra relacionado con la confianza que los usuarios sienten hacia la marca representada, en este caso por las universidades bogotanas. Dicha confianza es influenciada por el lazo que han creado los estudiantes, administrativos y graduados hacia las mismas. Para futuras investigaciones relacionadas, se sugiere abordar los atributos de la marca en una población mucho más amplia, para tener un mayor ámbito de validez, tomando como referente los hallazgos obtenidos en el presente estudio, de manera que pueda construirse un estudio longitudinal.

Palabras clave: Amor; Intimidad; Lovemarks; Marca; Misterio; Respeto.

\begin{abstract}
Lovermark or brand love, has been a term that has become relevant in recent years in the field of marketing, hence it is considered important to study it in various contexts This article aims to evaluate the attributes of the Lovemarks in Bogotá. For this, a deductive approach was used, under a quantitative research modality. The population was represented by 198 students, 9 administrative, 9 teachers and 25 graduates from different universities in Bogotá. An open and
\end{abstract}


closed question survey was used as the data collection instrument. This survey showed that the attribute "respect" was the one that obtained a more favorable Path coefficient with respect to "love", followed by "intimacy, "mystery" and finally the attribute "sensuality". Concluding that the love mark is related to the confidence that users feel towards the brand, represented in this case by Bogotá universities, which is influenced by the bond created by students, administrators and graduates towards them. For future research it is suggested to address the attributes of the brand in a much wider population are addressed, to have a greater scope of validity, taking as reference the findings obtained in the present study, so that a longitudinal study can be constructed.

Keywords: Love; Intimacy; Lovemarks; Brand; Mystery; Respect.

\section{Introducción}

Lovemarks o marcas amadas, es un concepto relevante para el mercadeo, tanto para profesionales como para académicos; su exponente principal es Roberts (2004), quien expresa algunos ejemplos de Lovemarks, como lo son: Real Madrid, Barbie, el musical Annie, Topper, entre otros.

Las variables que inciden en las Lovemarks son: amor, respeto, misterio, sensualidad e intimidad (Roberts, 2004). En este sentido, es importante tener presente que el amor de marca puede desarrollarse no solo frente a un bien o servicio como tal, sino también frente a instituciones representativas, tales como: universidades, sitios culturales, equipos deportivos, hospitales, u otros (Sarkar et al., 2016).

En este contexto, una gran cantidad de investigadores han desarrollado estudios enfocados en el análisis de las marcas (Echeverri y Rosker, 2011) y estas variables; tal es el caso de Carroll \& Ahuvia (2006), quienes, en el marco de una nueva construcción de marketing, evalúan el apego emocional apasionado, de los consumidores satisfechos, a marcas particulares, es decir, el amor de marca. Obteniendo que el amor de los consumidores satisfechos es mayor para las marcas en categorías de productos percibidas como más hedónicas (en comparación con las utilitarias), y para las marcas que ofrecen más en términos de beneficios simbólicos. Se obtuvo, también, que el amor a la marca está vinculado a niveles más altos de lealtad a esta y al voz a voz positivo. Los 
resultados también sugieren que los consumidores satisfechos tienden a ser menos leales a las marcas en categorías de productos más hedónicos, y a participar en un voz a voz más positivo sobre las marcas auto expresivas.

Por su parte, Batra et al. (2012), al investigar la naturaleza y las consecuencias del amor de marca, argumentan que la investigación sobre el amor por la marca debe basarse en una comprensión de cómo los consumidores realmente experimentan este fenómeno. A través de un estudio cualitativo, determinaron diferentes elementos o características del prototipo del amor por la marca del consumidor, y a través del modelado de ecuaciones estructurales en datos de encuestas obtuvieron que un modelo de orden superior produce siete elementos centrales: integración de marca propia, comportamientos impulsados por la pasión, conexión emocional positiva, relación a largo plazo, valencia de actitud general positiva, certeza de actitud y confianza (fortaleza) y angustia de separación anticipada.

Además de estos siete elementos centrales del amor de marca, el prototipo incluye creencias de calidad, como antecedente del amor de marca y la lealtad de marca, el voz a voz y la resistencia a la información negativa como resultados. Tanto el modelo de amor de marca de primer orden como el de orden superior predicen mejor la lealtad, el voz a voz y la resistencia, y proporcionan una mayor comprensión, que una medida general resumida del amor de marca (Batra et al. 2012).

Actualmente, dado el amplio espectro del mercado, competitividad, herramientas de marketing, innovación, entre otros factores claves para el desarrollo y posicionamiento de una marca, la meta de cualquier empresa o institución es tener una marca con tal resonancia y trascendencia que se considere una de las lovemarks, de manera que su presencia en el mercado transcienda en el tiempo, logrando un aumentativo posicionamiento de marca hasta llegar a la fidelización (lovemarks), debido a los lazos emocionales que desarrollen los consumidores respecto a las mismas.

El contexto universitario colombiano, en los últimos años, ha tenido una dinámica cambiante, no solo en aspectos académicos, sino en cuanto al número de estudiantes matriculados. Según datos reportados por el Sistema Nacional de Información de la Educación Superior -SNIES(2017), en el año 2017 el número de estudiantes matriculados, en al ámbito nacional, fue de 2.446.314, y en el 2018 de 2.408.041, presentándose una disminución de 38.273, que representa 
un 1,5\%. Esta situación ha despertado alarmas en el sector; es por ello por lo que las instituciones de educación superior (IES), como cualquier otra empresa, deben buscar posicionarse como marca y generar diferenciales entre ellas (Gabalán -Coello y Vásquez-Rizo, 2019; L. Montoya et al., 2019); toda vez que allí se indica el cómo el uso de este tipo de escalas permite contribuir a la gestión de marca, y gestionar los programas de fidelidad, de manera tal que se logre conectar con los diferentes públicos que responden al ámbito universitario, pensándolos como consumidores.

En ese sentido, la investigación desarrollada buscó analizar las lovemarks en el contexto universitario en Bogotá, tomando en consideración los puntos de vista de estudiantes, administrativos, docentes y graduados de diferentes universidades de Bogotá, entre ellas algunas acreditadas. Para ello, se identificaron los atributos de las Lovemarks, luego se calcularon las trayectorias de las variables independientes: respeto, misterio, sensualidad e intimidad, frente a la variable dependiente: amor; y, finalmente, para así evaluar los atributos de las Lovemarks: respeto, misterio, sensualidad e intimidad, en la generación de amor a la marca, para el contexto de las universidades en Bogotá.

Lo anterior, de manera que se pueda obtener una visión general, desde la perspectiva de la población objetivo, sobre los alcances que han tenido las referidas instituciones en su propósito de convertirse en una de las lovemarks. Con base en lo expuesto, se presenta la siguiente pregunta de investigación, la cual buscará ser respondida al finalizar el estudio: ¿Cuál es el nivel de influencia que tienen los atributos de la marca: respeto, misterio, sensualidad e intimidad, en la generación de amor a la marca, en las universidades en Bogotá?

\section{Revisión de literatura}

En este apartado, se presenta literatura relevante que expresa las posturas teóricas respecto al problema planteado, en función de las variables bajo estudio: amor, como variable dependiente, y los atributos de las Lovemarks, que son el respeto, intimidad, misterio y sensualidad.

En consecuencia, es relevante indicar que las denominadas Lovemarks son marcas que gozan de una lealtad más allá de la razón; estas no son propiedad de los fabricantes, productores ni de las empresas, pertenecen a la gente que las ama; además, los atributos que las caracterizan son: misterio, sensualidad e intimidad (Roberts, 2004). En este sentido, se destaca que el 
desarrollar amor por la marca, involucra elementos como: el sentido de pertenencia, aprecio y orgullo por la marca.

Adicionalmente, es importante señalar que las marcas desempeñan un rol como constructores de relaciones consumidor-marca, lo que involucra factores como el amor de marca, el gusto de marca, el odio de marca, la aversión de marca y la indiferencia de marca; así lo evidencia el estudio de Fetscherin et al. (2019).

\section{Atributos de una marca amor (Lovemarks)}

En líneas generales, el amor por la marca u otro referente surge a partir de las conexiones emocionales que es capaz de tocar la sensibilidad de los individuos profundamente. En este contexto, lovemarks "es la relación de contemplación, adoración y amor entre una marca y el comprador. Aquella marca por la cual los consumidores se desviven, por la que están dispuestos a muchos esfuerzos con tal de tenerla cerca y adorarla" (Delgado, 2014, p. 32).

En este sentido, se tiene que, para medir el amor a la marca, es necesario considerar los atributos de esta, a saber: respeto, intimidad, misterio y sensualidad (Roberts, 2004). En el contexto de las universidades bogotanas, estos atributos se ven reflejados en factores como el bienestar universitario, becas, auxilios financieros, calidad docente y educativa, ambiente educativo, infraestructura, entre otros.

Respeto

Esta dimensión obedece a la coherencia de la marca al cumplir sus promesas en cada contacto con el público objetivo; en este escenario se señala que "rendir al máximo es un mero requisito mínimo" (Roberts, 2004, p. 61). No obstante, en el contexto de esta investigación, se podría plantear que el respeto por la marca (universidades) involucra elementos como: credibilidad, actuación coherente, reputación y confianza que transmite la marca, toda vez que cada uno de estos, en el marco de las universidades colombianas, se refleja en factores como el valor de la matrícula, calidad académica y cumplimiento de planificaciones semestrales (Ariza et al., 2017). 


\section{Intimidad}

El amor hacia una marca también se encuentra relacionado con la intimidad, la misma "se relaciona con conceptos como empatía, compromiso a lo largo del tiempo y pasión” (Delgado, 2014, p. 36). A este respecto, se destaca que "la intimidad es el reto de nuestro tiempo. Exige tiempo y sentimientos sinceros, y ambas cosas son bienes muy escasos. De cómo las empresas obsesionadas con la intimidad crean empatía, compromiso y pasión” (Roberts, 2004, p. 8). En este escenario, es importante que las instituciones educativas, como es el caso de las universidades bogotanas, desarrollen mecanismos que permitan crear este tipo de vínculos con sus estudiantes y grupos de interés, para poder cumplir con este atributo de una marca amor (Serna et al., 2019).

\section{Misterio}

El misterio es la capacidad de la marca por emocionar en cada contacto con el público objetivo, generando una sensación permanente de novedad; está en las historias que cuanta la marca, sus asociaciones y los símbolos o representaciones que son ícono para el consumidor (Roberts, 2004). Asimismo, “el misterio lo componen las grandes historias que hay detrás de las grandes marcas, su capacidad para hacer soñar, los mitos e iconos que llegan a crear y la fuente de inspiración que suponen estas marcas para sus clientes” (Coello y Rodríguez, 2016, p. 20).

\section{Sensualidad}

Este atributo "se entiende como la capacidad de la marca de mantener los cinco sentidos a flor de piel" (Delgado, 2014, p. 35). También, se define como "seducción a través de los sentidos" (Hill, 2010, p. 86). A este respecto, se apunta que "las emociones primarias son breves, intensas e incontrolables, pero son las más complejas, pues combinan la cabeza y el corazón" (Roberts, 2004). En este orden de ideas, es relevante considerar que,

En el mundo sensorial, la carrera permanente a la búsqueda de los más rápido, lo más brillante o lo más sonoro, se agota en si misma muy pronto. La gente desconecta y la 
pierdes. Las Lovemarks necesitan Sensualidad, pero la necesitan con un toque humano. (Roberts, 2004, p. 108)

\section{Estrategia metodológica}

En esta sección se describen los aspectos metodológicos que guiaron la realización del estudio para dar respuesta a la hipótesis planteada, en función de las variables de estudio.

\section{Enfoque metodológico}

En el contexto de esta investigación, se tomó como referente el enfoque denominado "cebolla de investigación" de Saunders et al. (2007), en el cual se va desde la capa externa a la capa interna de la cebolla. Allí se establecen seis capas o etapas, estas son: filosofía de investigación, enfoque de investigación, estrategias de investigación, opción de investigación, horizonte de tiempo de investigación y elección de metodología de recopilación de datos.

La capa más externa es la filosofía de investigación, que establece el escenario para el proceso de investigación y define el método que se adopta como enfoque de investigación en el segundo paso. En el tercer paso, selecciona si se tendrá un único método o un sistema mixto según la naturaleza y complejidad del problema de investigación; mientras que, en el cuarto se adopta la estrategia de investigación, y la quinta capa identifica el horizonte temporal. El sexto paso representa la etapa en la que se establecen los mecanismos para la recopilación de datos. Los beneficios de la cebolla de investigación son, por tanto, que en primera instancia crea una serie de etapas bajo las cuales se pueden entender los diferentes métodos de recopilación de datos, y en segunda ilustra los pasos por los cuales se puede describir un estudio metodológico (Saunders et al., 2007).

En el contexto de la investigación desarrollada, la filosofía de investigación implementada fue positivismo, la cual plantea preguntas e hipótesis de investigación que pueden evaluarse y analizarse (Tonuchi, 2019). Derivado de ello, el enfoque de investigación utilizado fue el deductivo, el cual desarrolla hipótesis sobre una teoría preexistente, y luego formula el enfoque de investigación para probarla (Silverman, 2013). El enfoque deductivo puede considerarse 
particularmente adecuado para el enfoque positivista, que permite la formulación de hipótesis y la prueba estadística de los resultados esperados a un nivel aceptado de probabilidad (Snieder \& Larner, 2009).

En cuanto a la tercera etapa, la estrategia de investigación es aquella que describe cómo el investigador pretende llevar a cabo el trabajo (Saunders et al., 2007). La estrategia puede incluir varios enfoques diferentes; en el caso del presente estudio, se hizo uso de encuestas y revisión sistemática de la literatura.

Posteriormente, la cuarta capa de la cebolla de investigación, conocida como opción de investigación, ayuda a saber al investigador si está bien combinar tanto la metodología cuantitativa como la cualitativa, o utilizar solo una metodología. Según Saunders et al. (2007), hay tres opciones resumidas en la cebolla de investigación que incluyen la opción o el enfoque de investigación de métodos mono, mixto y múltiple.

Con respecto a la investigación que se está desarrollando, se utilizó el método cuantitativo, el cual "utiliza la recolección de datos para probar hipótesis con base en la medición numérica y el análisis estadístico, con el fin establecer pautas de comportamiento y probar teorías" (Fernández Collado et al., 2014, p. 4). En este contexto, se analizará el amor a la marca que tienen los encuestados hacia las universidades en Bogotá donde realizaron estudios. Al mismo tiempo, se empleará un análisis estadístico a través del programa SMARTPLS3®., para establecer los Path coeficients (coeficientes de ruta o trayectoria) de cada una de las variables independientes establecidas, con respecto al amor de marca.

Seguidamente, se tiene que la quinta etapa constituye el horizonte de tiempo de investigación; el horizonte temporal describe el tiempo requerido para la finalización del trabajo del proyecto. En la cebolla de investigación se especifican dos tipos de horizontes temporales: el transversal y el longitudinal (Bryman, 2012). Para efectos de esta investigación, se considerará el horizonte temporal de la sección transversal, el cual se utiliza cuando la investigación se refiere al estudio de un fenómeno particular en un momento específico, tal como se realiza el estudio en función de la opinión, de la población bajo estudio, con respecto a las universidades colombianas como lovemarks.

Finalmente, la sexta etapa es la elección de metodología de recopilación y el análisis de datos, que dependen del enfoque metodológico utilizado (Bryman, 2012). Esta capa explica cómo 
se recopilan y analizan los datos utilizados en la investigación. También, menciona la fuente de datos, el diseño de la investigación, la muestra, el tamaño de la muestra, la ética de la muestra, las limitaciones de la muestra, la fiabilidad y la validez de la investigación; en las secciones siguientes del artículo se realizará la explicación de cada uno de estos puntos.

\section{Población y muestra}

La muestra estuvo representada por 198 estudiantes, 9 administrativos, 9 docentes y 25 graduados de diferentes universidades de Bogotá, entre ellas algunas acreditadas. Es relevante indicar que la estrategia que se utilizó para el muestreo fue a conveniencia, toda vez que no se contaba con un marco muestral que permitiera realizar un proceso probabilístico. En la tabla 1 se ilustra la distribución demográfica de la muestra:

\section{Tabla 1}

Distribución de la muestra

\begin{tabular}{lll}
\hline Rol desempeñado & No & Porcentaje \\
\hline Administrativo & 9 & $4 \%$ \\
\hline Docente y profesor & 9 & $4 \%$ \\
\hline Estudiantes & 198 & $82 \%$ \\
\hline Graduados & 25 & $10 \%$ \\
\hline Total & 241 & $100 \%$ \\
\hline
\end{tabular}

Nota: Elaboración propia

\section{Variables}

1. Variable dependiente: Amor a la marca

2. Variables independientes: Respeto, intimidad, misterio y sensualidad. 


\section{Hipótesis}

H1: Los atributos de la marca: respeto, intimidad, misterio y sensualidad, influyen en la percepción de los estudiantes, administrativos, docentes y graduados hacia las universidades en Bogotá, generando amor hacia la marca que representan las mismas, convirtiéndolas en una de las lovemarks.

H1a: El amor a la marca (Lovemarks) de las universidades en Bogotá se ve influenciado por el respeto.

H1b: El amor a la marca (Lovemarks) de las universidades en Bogotá se ve influenciado por el misterio.

H1c: El amor a la marca (Lovemarks) de las universidades en Bogotá se ve influenciado por la intimidad.

H1d: El amor a la marca (Lovemarks) de las universidades en Bogotá se ve influenciado por la sensualidad.

\section{Instrumentos de recolección de datos}

Los instrumentos de recolección de datos forman parte de la estrategia de investigación; esta describe cómo el investigador pretende llevar a cabo el trabajo (Saunders, 2007). Para efectos del presente estudio, se utilizó como estrategia la encuesta, toda vez que este mecanismo tiende a usarse en proyectos de investigación cuantitativa e implica tomar muestras de una proporción representativa de la población (Bryman y Bell, 2011). La estrategia de la encuesta se usa principalmente para observar variables contribuyentes entre diferentes datos. En este orden de ideas, se diseñó una encuesta de preguntas abiertas y cerradas; estas últimas con 5 alternativas de repuesta: 5= Totalmente de acuerdo; $4=$ Parcialmente de acuerdo; $3=\mathrm{Ni}$ acuerdo ni en desacuerdo; $2=$ Parcialmente en desacuerdo; $1=$ Totalmente en desacuerdo.

Amor: fue medida con 9 ítems de respuesta cerrada, los cuales son "Esta marca es especial para mí", "Esta marca me causa un asombro", "Esta marca es totalmente increíble", "Esta marca me hace sentir bien", "Esta es una marca maravillosa", "Aprecio esta marca por todo lo que me ha 
brindado", "Tiene sentido preferir esta marca en lugar de cualquier otra", "A esta marca la prefiero, incluso si hay otras parecidas" y "Definitivamente es inteligente preferir esta marca".

Respeto de la marca: fue medida con 9 ítems de respuesta cerrada, donde se encuentran "Considero que es una marca de calidad", "Esta marca es sincera para mí", "Esta marca es comprometida para mí", "Esta marca se comunica conmigo", "Esta marca es honesta para mí”, "Puedo confiar en esta marca", "Esta mara es responsable para mí", "Considero que soy fiel a esta marca" y "La marca posee un alto desempeño".

Misterio: fue medida con 7 ítems de respuesta cerrada, dando lugar a "Esta marca agrega experiencia a mi vida", "Esta marca despierta buenos recuerdos en mí", "Esta marca captura la sensación de mi vida", "La marca cuenta con una historia que genera emoción", "La marca ha sido inspiradora en mi vida", "Esta marca es parte de mi vida" y "Si la marca desapareciera, mi vida cambiaría mucho"

Sensualidad: fue medida con 8 ítems de respuesta cerrada, los cuales son "El diseño de los anuncios de esta marca está muy bien hecho", "Las actividades que programa esta marca me atraen", "Cuando voy a otras ciudades en donde hay sedes de esta marca las visito", "El diseño del sitio web para esta marca es perfecto", "Me atrae entrar a las edificaciones de esta marca", "Las imágenes de esta marca tienen un hermoso esquema de colores", "Esta marca tiene una publicidad increíble" y "Es una marca atractiva o agradable".

Intimidad: fue medida con 8 ítems de respuesta cerrada, donde se encuentran "La marca se preocupa por mis intereses", "Me siento conectado con esta marca", "La marca establece canales de comunicación eficientes para mis opiniones", "Recomendaría esta marca a amigos", "Me siento orgulloso(a) de esta marca", "Esta marca genera relaciones cercanas conmigo", "He pensado Que siempre me gustaría estar conectado con la marca" y "Considero que las decisiones que adopta la marca buscan beneficiarme".

\section{Validación de contenido y consideraciones éticas}

El instrumento de recolección de datos fue validado de acuerdo con el criterio de expertos, quienes verificaron la congruencia de los ítems o preguntas, en función de cada una de las variables estudiadas. Por otro lado, para la aplicación de la encuesta se realizó un consentimiento informado 
a los encuestados, y todos los cuestionarios se realizaron de forma anónima, con fines exclusivamente académicos.

\section{Proceso de análisis de datos}

En primer lugar, se establecieron las hipótesis con respecto a la variable independiente y dependientes del estudio, en el marco de un enfoque deductivo. Después de ser validado el instrumento, se implementó en la plataforma de Google formularios, informando a los encuestados que, al participar, se autorizaba el tratamiento de su información, por parte de los investigadores. Dicha información tendría un procesamiento anónimo y agregado. Igualmente, para cualquier inquietud, se les facilitaron los datos de contacto de los investigadores. En la tabla 2 se muestran los parámetros más relevantes de la recolección de datos.

\section{Tabla 2}

Parámetros más relevantes de la recolección de datos

\begin{tabular}{ll}
\hline Parámetro & Datos \\
\hline Total de preguntas & 57 \\
\hline Preguntas obligatorias & 57 \\
\hline Preguntas no obligatorias & 0 \\
\hline Preguntas cerradas & 49 \\
\hline Preguntas abiertas & 8 \\
\hline Total de respuestas & 241 \\
\hline
\end{tabular}

Nota: Elaboración propia

Una vez recopilados los datos, y teniendo en cuenta que el objetivo de la investigación es evaluar la relación entre las variables de respeto, intimidad, misterio y sensualidad, frente al amor, se hizo uso del modelado de ruta de mínimos cuadrados parciales (PLS), debido a que este método permite, a través de las técnicas de varianza, la validación de instrumentos y evalúa las relaciones entre constructos (Henseler et al., 2009).

Posteriormente, una vez establecido el modelo, se llevó a cabo la metodología planteada por Hair et al. (2011), para la evaluación de la fiabilidad de la consistencia interna y validez 
convergente del instrumento, a través de la verificación de los valores de aceptabilidad en la prueba de consistencia interna, para el coeficiente alpha de Cronbach, la fiabilidad compuesta y la varianza media extraída (AVE). Adicionalmente, se realizaron los análisis para la comprobación de la validez discriminante y, finalmente, la calidad del modelo, sus variables y sus ítems, a través del análisis de colinealidad, y de las relaciones finales entre las variables endógenas y exógenas, a través de los coeficientes de trayectoria del modelo, el coeficiente de determinación R2, el tamaño del efecto f2, la relevancia predictiva Q2 y el tamaño del efecto q2; todo lo anterior, a través del uso de SMARTPLS3®.

Para el uso del software SMARTPLS3®. se dispuso de tres procedimientos principales: Algoritmo PLS, Bootstrapping y Blindfolding. En primera instancia, el algoritmo PLS tiene aplicaciones predictivas, y generalmente es recomendado cuando existe poca información teórica sobre el tema. En segunda, el procedimiento de Bootstrapping, responde a un "tipo de técnica de remuestreo de datos que permite resolver problemas relacionados con la estimación de intervalos de confianza o la prueba de significación estadística” (Ledesma, 2016). En tercera instancia, el procedimiento Blindfolding, permite evaluar la relevancia predictiva del modelo, a través del cálculo de parámetros como Q2, el cual "postula que el modelo estructural debe ser capaz de predecir adecuadamente cada indicador del constructo latente endógeno" (Hair et al., 2011).

Con base en el modelo, y con los objetivos e hipótesis de la investigación definidos, los cuales fueron asociados en la construcción del cuestionario, se establece el modelo de la figura 1. 


\section{Figura 1}

Modelo de ruta PLS propuesto

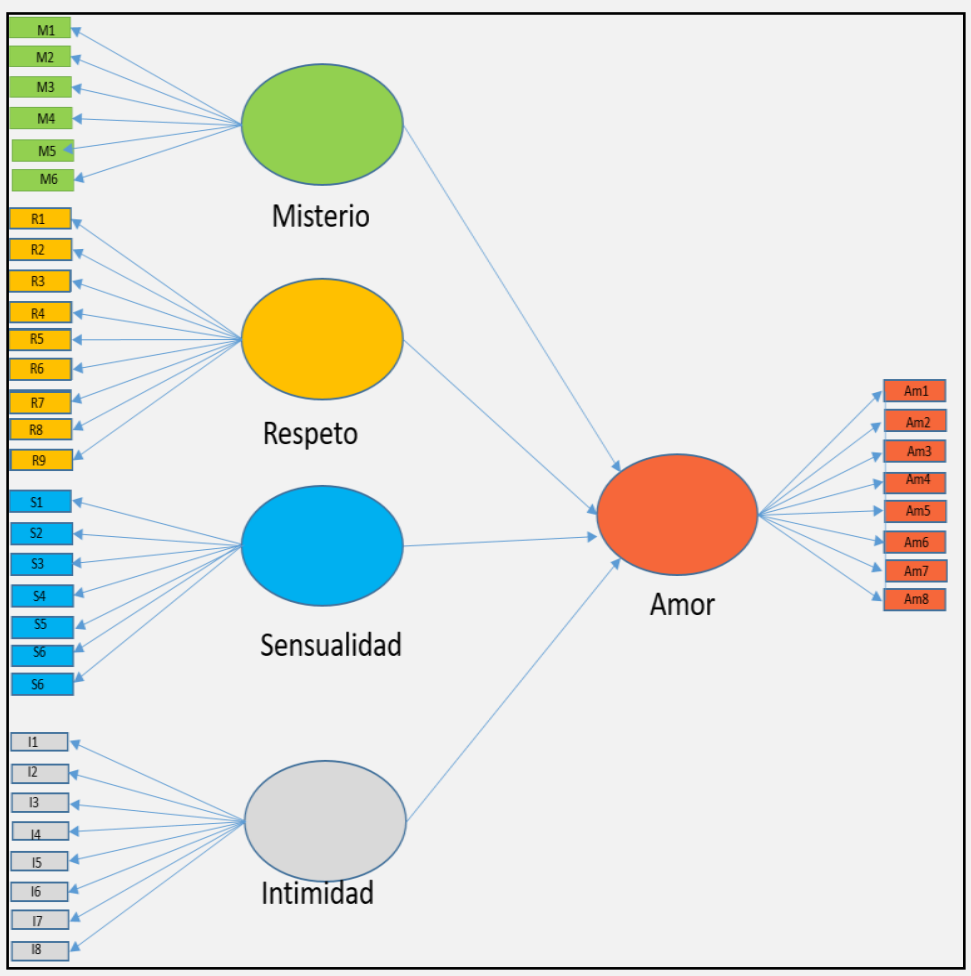

Nota: Elaboración propia

\section{Resultados}

A continuación, se presentan los resultados de la investigación, en función de los objetivos específicos del estudio. En primera instancia, se identifican los atributos de las Lovemarks, según los resultados de la validación empírica en el ámbito de la educación superior, se calculan los Path coefficients y se presenta lo referido a la comprobación de hipótesis.

\section{Identificación de los atributos de las marcas amor (lovemarks)}

El amor de marca, de acuerdo con la revisión de literatura realizada, cuenta con los siguientes atributos: misterio, respecto, sensualidad e intimidad. 
Cálculo de los Path coefficients, de las variables independientes: respeto, misterio, sensualidad e intimidad, frente a la variable dependiente: amor

Al analizar los resultados obtenidos de la encuesta aplicada, a la población de estudiantes, administrativos, graduados y profesores de las universidades bogotanas, a través del programa SMARTPLS3®. se obtuvieron los Path coefficients de las variables independientes en estudio: respeto, misterio, sensualidad e intimidad, frente a la variable central o dependiente: amor de marca. En la figura 2, se exponen los valores establecidos para cada una de ellas.

\section{Figura 2}

Path coefficients

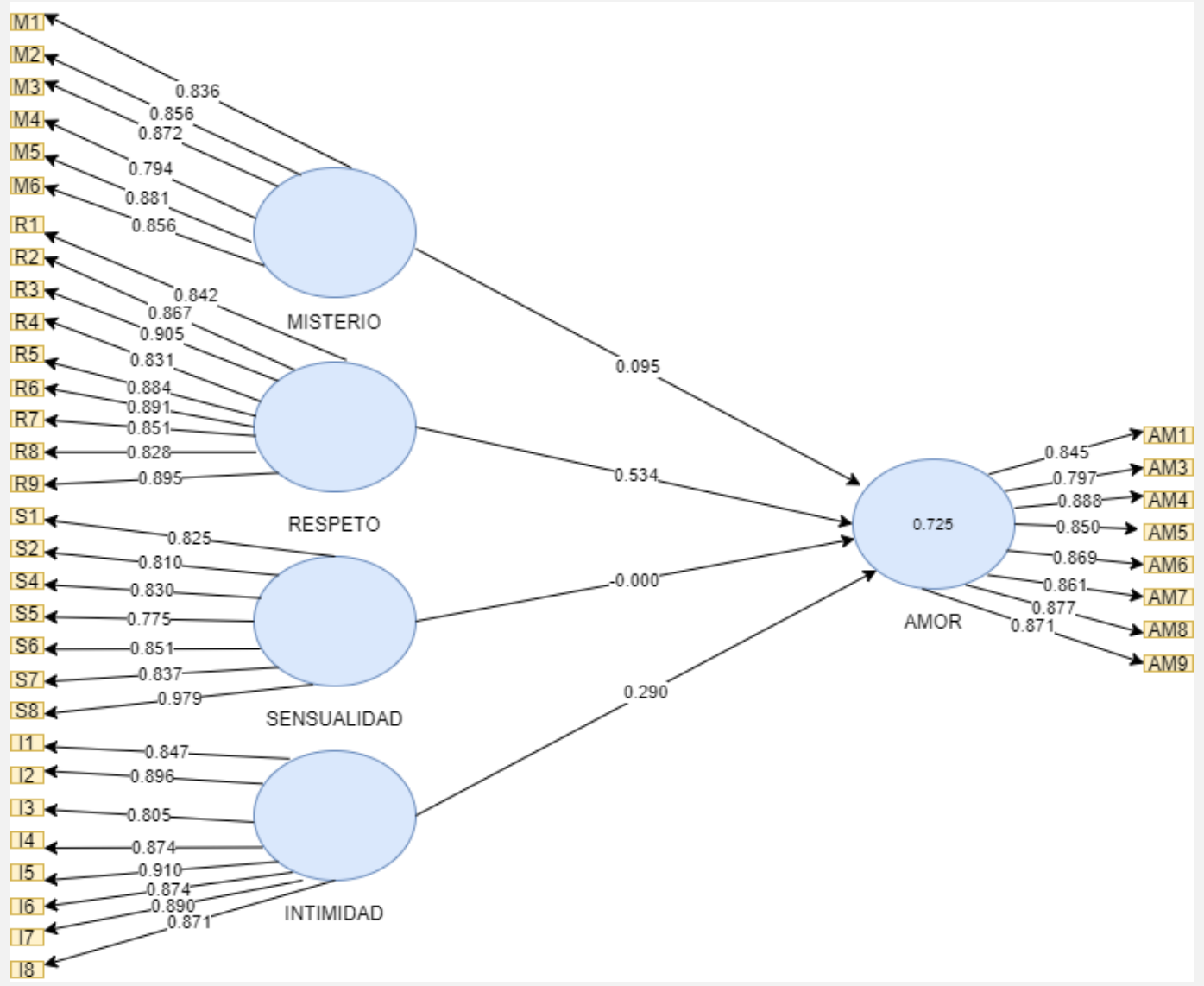

Nota: Elaboración propia. 
Nivel de influencia que tienen los atributos de la marca: respeto, misterio, sensualidad e intimidad, en la generación de amor a la marca, de las universidades en Bogotá

Tal como se aprecia en la figura 2, la variable "respeto" fue la que obtuvo un Path coefficient más favorable, con respecto al "amor", alcanzando un valor de 0,534 . Dicho coeficiente indica el efecto directo de la variable "respeto", que constituye una causa de la variable "amor", que es un efecto directo de la primera.

Con respecto a la variable de "intimidad", el Path coefficients alcanzado fue de 0,290, ubicándola luego del "respeto". Lo cual implica que también constituye una causa principal del desarrollo de amor por la marca.

Al mismo tiempo, de acuerdo con lo representado en la figura 2, la variable "misterio" obtuvo un coeficiente de 0,095, frente a la variable "amor". Esto refleja que su nivel de influencia en el "amor" de marca, es menor que las anteriores.

Finalmente, la variable sensualidad obtuvo un coeficiente negativo de $-0,000$, frente a la variable "amor", lo que indica que su influencia en la percepción y el amor a la marca de los universitarios encuestados es nula.

Todos los coeficientes arrojados para cada una de las variables bajo estudio se obtuvieron de la muestra original (sample), por medio de su análisis en el programa SMARTPLS3®., y se estimaron en función de la muestra de 198 estudiantes, 9 administrativos, 9 docentes y 25 graduados.

\section{Atributos de las Lovemarks en el ámbito de la educación superior}

La tabla 3, a continuación, presenta los resultados y soporte a las hipótesis iniciales planteadas; es relevante indicar que se utilizó el proceso de Bootstrapping para tal fin, y se logró con él soportar las hipótesis H1a y H1c, siendo en ellas la intimidad y el respeto las dos variables influyentes en el constructo Amor o Lovemarks en el ámbito de la educación superior. 
Tabla 3

Resultados del Bootstrapping

\begin{tabular}{|c|c|c|c|c|c|}
\hline & $\begin{array}{c}\text { Original } \\
\text { Sample (O) }\end{array}$ & $\begin{array}{c}\text { Sample Mean } \\
\text { (M) }\end{array}$ & $\begin{array}{l}\text { Standard } \\
\text { Deviation } \\
\text { (STDEV) }\end{array}$ & $\begin{array}{c}\text { T Statistics } \\
(|\mathrm{O} / \mathrm{STDEV}|)\end{array}$ & P Values \\
\hline $\begin{array}{l}\text { INTIMIDAD } \\
\text {-> AMOR }\end{array}$ & 0,290 & 0,293 & 0,082 & 3,519 & 0,000 \\
\hline $\begin{array}{l}\text { MISTERIO - } \\
\text { > AMOR }\end{array}$ & 0,095 & 0,094 & 0,068 & 1,395 & 0,164 \\
\hline $\begin{array}{l}\text { RESPETO -> } \\
\text { AMOR }\end{array}$ & 0,534 & 0,533 & 0,072 & 7,440 & 0,000 \\
\hline $\begin{array}{l}\text { SENSUALID } \\
\text { AD -> AMOR }\end{array}$ & 0,000 & $-0,002$ & 0,067 & 0,002 & 0,998 \\
\hline
\end{tabular}

Nota: elaboración propia

\section{Discusión}

Los hallazgos de la revisión de literatura muestran que los atributos de las lovemarks son el respeto, la intimidad, el misterio y la sensualidad (Roberts, 2004); no obstante, para el ámbito de la educación superior en Colombia, la evidencia empírica muestra que las dimensiones que explican consistentemente el constructo amor a la marca (Lovemarks) en las universidades bogotanas es el respeto y la intimidad.

Tal como se pudo evidenciar, el amor de la marca se encuentra relacionado con la confianza que los usuarios sienten hacia la marca, la cual se ve influenciada por el lazo que ha creado la comunidad académica hacia las mismas; compromiso este que ha sido desarrollado a lo largo del tiempo. En función de esto, se destaca que, tal como apunta Roberts (2004), "las lovemarks necesitan Sensualidad, pero la necesitan con un toque humano” (p. 108).

En concordancia con lo anterior, Fetscherin et al. (2019), encontraron que las marcas desempeñan un rol como constructores de relaciones consumidor-marca, lo que involucra factores como el amor de marca, el gusto de marca, el odio de marca, la aversión de marca y la indiferencia de marca.

En líneas generales, se logró identificar los atributos de las lovemarks, calcular los Path coefficient de las variables independientes (atributos), con respecto a la variable dependiente 
(amor), para finalmente establecer el nivel de influencia que tienen los atributos de la marca: respeto, misterio, sensualidad e intimidad, en la generación de amor a la marca, de las universidades en Bogotá, dando cumplimiento a los objetivos planteados. Metodológicamente, no se presentaron limitaciones para el análisis de datos y logro de los propósitos establecidos al inicio del estudio.

En este contexto, el nivel de influencia que tienen los atributos de la marca: respeto, misterio, sensualidad e intimidad, en la generación de amor a la marca, de las universidades en Bogotá, es significativo, especialmente en cuanto al respeto que desarrollan los estudiantes, administrativos, docentes y graduados frente a las instituciones donde cursaron estudios y lograron obtener un título profesional. Como complemento a este planteamiento, se destaca lo expuesto por Roberts (2004), quien apunta que el respeto nace de lo que hacemos, del cumplimiento o no de lo que se promete en cada interacción con el público objetivo.

En cuanto al ejercicio del marketing, según los resultados del trabajo de investigación, para las universidades bogotanas la construcción de su imagen como una marca amada (lovemarks) está asociada principalmente al respeto que poseen los estudiantes, administrativos, docentes y graduados hacia las instituciones, el cual está relacionado con la sinceridad, compromiso, honestidad percibida, confianza inspirada, fidelidad, desempeño de esta, y su percepción por parte de los estudiantes.

Asimismo, se destaca que, en una investigación reciente, enfocada en el análisis de las diferencias entre estudiantes y egresados en un estudio de "marca de amor" en una universidad pública en Colombia, "los resultados validaron los efectos positivos de las variables amor por la marca, experiencia de marca y participación de la marca sobre la lealtad hacia ella, un factor determinante de lovemarks para una universidad" (I. Montoya et al., 2020, p. 43). En relación con el referido estudio, la presente investigación posee una ventaja comparativa, puesto que engloba una población más amplia, de múltiples universidades colombianas, mientras que el citado documento solo se enfocó en el amor de marca hacia la Universidad Nacional de Colombia (UNAL).

En suma, este estudio complementa ejercicios empíricos previos en Colombia (Ariza et al., 2017; Serna et al., 2019), en tanto que aborda el marketing en el ámbito de la educación superior, en aras de dar cuenta de las prácticas, acciones, tácticas y estrategias de marketing que configuran 
el valor percibido y la construcción de marca desde los grupos de interés de las instituciones de educación superior.

\section{Conclusiones}

Es relevante indicar que el marketing es un factor importante en el desarrollo de las lovemarks; de las estrategias establecidas a través del ejercicio del mismo, dependerá en gran medida el nivel de competitividad de las marcas y su posicionamiento, creando lazos con los usuarios, asociados con su identidad, género, edad, preferencias, valores, costumbres, entre otros aspectos de interés que deben considerarse para el desarrollo del amor de marca, que es el objetivo central de los equipos de marketing en cualquier tipo de empresa o institución.

En consecuencia, se sugiere continuar con estudios que reconozcan con mayor claridad las necesidades de los grupos de interés - estudiantes, profesores, egresados, padres de familia, empresas, etc. - de este tipo de instituciones, para así generar propuestas de valor dirigidas a ellos desde las universidades, que apliquen modelos de marketing adaptados a las dinámicas y naturaleza de este tipo de instituciones.

Finalmente, para las futuras investigaciones que aborden la temática de las lovemarks en las universidades bogotanas, se sugiere abordar los atributos de la marca en una población mucho más amplia, para tener un mayor ámbito de validez, tomando como referente los hallazgos obtenidos en el presente estudio, de manera que pueda construirse un estudio longitudinal, que ofrezca una perspectiva y contrastación de los hallazgos mucho más desarrollada.

\section{Referencias}

Ariza, R., Rojas, S., y Robayo, O. (2017). Comparación de las prácticas de gestión comercial en las instituciones de educación superior a partir de las diferencias entre las percepciones de directores de mercadeo y admisión y del potencial estudiante en proceso de elección de una carrera profesión. Libre Empresa, 14(2), 13-31. https://doi.org/10.18041/16572815/libreempresa.2017v14n2.1594 
Batra, R., Ahuvia, A., \& Bagozzi, R. P. (2012). Brand love. Journal of Marketing, 76(2), 1-16. https://doi.org/10.1016/j.aebj.2015.04.001

Bryman, A. (2012). Social research methods (5 Ed.). Oxford University Press.

Bryman, A., \& Bell, E. (2011). Business Research Methods. OUP Oxford.

Carroll, B. A., \& Ahuvia A. C. (2006). Some antecedents and outcomes of brand love. Marketing Letters, 17(2), 79-89. https://doi.org/10.1007/s11002-006-4219-2

Coello, O., y Rodríguez, M. G. (2016). Relación de una marca con sus consumidores según el concepto de lovemark. Caso: Nestea (Trabajo de grado de pregrado). Universidad Católica Andrés Bello. http://biblioteca2.ucab.edu.ve/anexos/biblioteca/marc/texto/AAT2421.pdf

Delgado, P. (2014). Elementos de identidad de marca vs elementos de lovemarks (Trabajo de grado de pregrado). Universidad Católica Andrés Bello. http://biblioteca2.ucab.edu.ve/anexos/biblioteca/marc/texto/AAS7351.pdf

Echeverri, L., y Rosker, E. (2011). Diferencias en la construcción de marca país: Canadá y Colombia. Revista Virtual Universidad Católica del Norte, (33), 187-212. https://revistavirtual.ucn.edu.co/index.php/RevistaUCN/article/view/18/36

Fetscherin, M., Guzman, F., Veloutsou, C., \& Cayolla R. (2019). Latest research on brand relationships: introduction to the special issue. Journal of Product and Brand Management, 28(2), 133-139. https://doi.org/10.1108/JPBM-12-2018-2167

Fernández Collado, C., Baptista Lucio, P., y Hernández Sampieri, R. (2014). Metodología de la Investigación. McGraw Hill.

Gabalán-Coello, J. y Vásquez-Rizo, F. E. (mayo-agosto, 2019). Noción de calidad profesoral desde la percepción estudiantil. Revista Virtual Universidad Católica del Norte, (57), 24-39. https://doi.org/10.35575/rvucn.n57a3

Hair, J., Ringle, C. M., \& Sarstedt, M. (2011). PLS-SEM: Indeed a Silver Bullet. Journal of Marketing Theory and Practice, 19(2), 139-152. https://doi.org/10.2753/MTP1069$\underline{6679190202}$

Henseler, J., Ringle, C. M., \& Sinkovics, R. (2009). The use of partial least squares path modeling in international marketing. Advances in International Marketing, (20), 277-319. https://doi.org/10.1108/S1474-7979(2009)0000020014

Hill, D. (2010). Emotionomics: Leaveraging emotions for business success (2 Ed.). KoganPage. 
Ledesma, R. (2016). Introducción al Bootstrap: Desarrollo de un ejemplo acompañado de software de aplicación. Tutorials in Quantitative Methods for Psychology, 4(2), 51-60. https://doi.org/10.20982/tqmp.04.2.p051

Montoya, I., Sánchez, J., Rojas, S., \& Montoya, A. (2020). Lovemark Effect: Analysis of the Differences Between Students and Graduates in a Love Brand Study at a Public University. Innovar, 30(75), 43-56. http://dx.doi.org/10.15446/innovar.v30n75.83256

Montoya, L., Sánchez, J., Rojas, S., Castaño. J., y Montoya, I. (2019). Percepción de una marca amor. Caso de la Universidad Nacional de Colombia. Económicas CUC, 40(2), 117-138. https://revistascientificas.cuc.edu.co/economicascuc/article/download/2062/2556?inline1

Roberts, K. (2004). El futuro más allá de las marcas: Lovemarks. Empresa activa. Saatchi \& Saatchi.

Sarkar, A., Sarkar, J. G., \& Gopal, K. S. (2016). How to develop emotional attachment amongst patients towards hospitals? A qualitative investigation in the context of emerging Indian market. Journal of Asia Business Studies, 10(3) 213-229. https://doi.org/10.1108/JABS$\underline{05-2015-0058}$

Saunders, M., Lewis, P., \& Thornhill, A. (2007). Research Methods for Business Students (6 Ed.) Pearson.

Serna, D., Rojas, S., y Robayo, Ó. (2019). Valor percibido en instituciones de educación superior por parte de estudiantes de modalidad virtual. Pensamiento y Gestión, (46), 79-107. http://rcientificas.uninorte.edu.co/index.php/pensamiento/article/view/10963

Silverman, D. (2013). Doing Qualitative Research: A practical handbook. Sage.

Sistema Nacional de Información de la Educación Superior -SNIES-. (2017). Matriculados: Información poblacional. https://hecaa.mineducacion.gov.co/consultaspublicas/content/poblacional/index.jsf

Snieder, R., \& Larner, K. (2009). The Art of Being a Scientist: A Guide for Graduate Students and their Mentors. Cambridge University Press.

Tonuchi, J. (2019). Analysis of Saunders Research Onion. Thesismind. https://thesismind.com/analysis-of-saunders-research-onion/ 